\title{
EFFECT OF TWO DIFFERENT DOSES OF INTRAVENOUS ESMOLOL AND LIDOCAINE PREMEDICATION ON STRESS RESPONSE DURING INTUBATION- MEASURED BY SERUM CORTISOL LEVELS
}

\author{
Viswanathan Karthik Narayanan', Gopal Deepa ${ }^{2}$
}

${ }^{1}$ Assistant Professor, Department of Anaesthesiology, Government Vellore Medical College Hospital, Adukkamparai, Vellore. ${ }^{2}$ Assistant Professor, Department of Anaesthesiology, Government Vellore Medical College Hospital, Adukkamparai, Vellore.

\section{ABSTRACT}

\section{BACKGROUND}

The aim is to study and compare the effect of two different doses of esmolol and lidocaine on the attenuation of stress response during intubation by measuring serum cortisol.1,2

\section{MATERIALS AND METHODS}

This study was a prospective randomised single blinded study conducted at Government Vellore Medical College. After obtaining ethical committee approval, 60 patients posted for elective general surgeries under general anaesthesia were randomised into three groups, with each having 20 patients. Group C- Control group receiving normal saline, Group L- Low dose group receiving Esmolol $1 \mathrm{mg} / \mathrm{kg}$ and Lidocaine $1.5 \mathrm{mg} / \mathrm{kg}$, Group H- High dose group receiving Esmolol $2 \mathrm{mg} / \mathrm{kg}$ and Lidocaine $2.5 \mathrm{mg} / \mathrm{kg} .{ }^{3}$ Drug volume of $10 \mathrm{~mL}$ is standardised in all 3 groups. Serum cortisol estimation was done in all the three groups before premedication with Esmolol and Lidocaine and 5 minutes after intubation. ELISA method was adopted for cortisol estimation.

\section{RESULTS}

According to this study, there was significant reduction in pulse rate, systolic and diastolic blood pressure, and serum cortisol level after intubation in high dose group when compared to low dose group and control group. ${ }^{4}$

\section{CONCLUSION}

Premedication with high doses of Esmolol and Lidocaine before intubation attenuates the stress response and thereby may decrease the morbidity and mortality in susceptible individuals.

\section{KEYWORDS}

Esmolol and Lidocaine, Cortisol, Stress Response.

HOW TO CITE THIS ARTICLE: Narayanan VK, Deepa G. Effect of two different doses of intravenous esmolol and lidocaine premedication on stress response during intubation- Measured by serum cortisol levels. J. Evolution Med. Dent. Sci. 2017;6(14): 1124-1128, DOI: $10.14260 / J e m d s / 2017 / 244$

\section{BACKGROUND}

General anaesthesia requires laryngoscopy and intubation to undergo surgeries.

Laryngoscopy and endotracheal intubation activate the neuroendocrine axis and cause the release of catecholamines and cortisol leading to increase in heart rate, blood pressure and myocardial oxygen demand.

Activation of sympathetic nervous system also produces coronary artery vasoconstriction which reduces the supply of oxygen to the myocardium causing myocardial ischaemia and dysrhythmias.

Intravenous and inhalation anaesthetic agents have no appreciable effects on these stress response. Lignocaine and esmolol has some proven effect on attenuation of stress response during laryngoscopy and endotracheal intubation. This is confirmed by the reduction in serum cortisol levels. Esmolol hydrochloride is a short acting beta-one selective adrenergic receptor blocker with a distribution half-life of 2

Financial or Other, Competing Interest: None.

Submission 09-01-2017, Peer Review 02-02-2017,

Acceptance 08-02-2017, Published 16-02-2017.

Corresponding Author:

Dr. Viswanathan Karthik Narayanan,

Assistant Professor,

Government Vellore Medical College Hospital,

Adukkamparai, Vellore-632011.

E-mail:karthik.narayanan73@gmail.com

DOI: $10.14260 /$ jemds $/ 2017 / 244$ minutes and an elimination half-life of 9 minutes. Esmolol is an ideal drug during a short-lived stress such as tracheal intubation.

Lidocaine is a class Ib membrane stabilising agent. It decreases airway sensitivity, depresses airway reflexes to tracheal intubation and suppresses cardiac dysrhythmias.5,6

Thus, to find out the optimum dose of esmolol and lidocaine in attenuating the intubation stress response, two different doses of these drugs have been compared. Serum cortisol estimation is done to prove the effect of these drugs on stress response.

\section{Aim of the Study}

To study and compare the effect of two different doses of esmolol and lidocaine on the attenuation of stress response during intubation by measuring serum cortisol.7,8

\section{MATERIALS AND METHODS}

This study was a prospective randomised single blinded study conducted at Government Vellore Medical College.

After obtaining ethical committee approval, 60 patients posted for elective general surgeries under general anaesthesia were randomised into three groups, with each having 20 patients.

Group C - Control group receiving normal saline.

Group L - Low dose group receiving Esmolol $1 \mathrm{mg} / \mathrm{kg}$ and Lidocaine $1.5 \mathrm{mg} / \mathrm{kg}$. 
Group H - High dose group receiving Esmolol $2 \mathrm{mg} / \mathrm{kg}$ and Lidocaine $2.5 \mathrm{mg} / \mathrm{kg}$.

Drug volume of $10 \mathrm{~mL}$ is standardised in all 3 groups.

Serum cortisol estimation was done in all the three groups before premedication with Esmolol and Lidocaine and 5 minutes after intubation. ELISA method was adopted for cortisol estimation.

\section{Inclusion Criteria}

- $\quad$ ASA I \& II Patients.

- Age 18-60 Years.

- Both sexes, patients undergoing elective general surgeries.

\section{Exclusion Criteria}

- History of pulmonary and cardiac disorder,

- Pregnancy,

- History of allergy to study drug,

- Medical illness like Hypertension, Diabetes mellitus and asthma.

- Impaired kidney or liver function,

- Anticipated difficult airway.

Written informed consent was obtained from the patients before surgery. In control group (Group C), intravenous cannula was secured and blood sample for cortisol was collected. Pulse Oximeter, non-invasive blood pressure and Electrocardiogram were established. Baseline pulse rate, SPO2 and blood pressure were recorded. $10 \mathrm{~mL}$ of normal saline was given during pre-oxygenation. Induction was done with $2 \mathrm{mcg} / \mathrm{kg}$ of fentanyl, $5 \mathrm{mg} / \mathrm{kg}$ of thiopentone and 2 $\mathrm{mg} / \mathrm{kg}$ of Scoline. 5 minutes after intubation, again the blood sample for cortisol was collected. At the same time, pulse rate, diastolic blood pressure, systolic blood pressure and SPO2 were noted.9,10 Patient was maintained with nitrous oxide, oxygen, fentanyl and atracurium in titrated doses. Intraoperatively vitals were stable. After adequate attempts, patient was reversed with neostigmine and glycopyrrolate and was extubated.

In Group L, intravenous cannula secured and blood sample for cortisol was collected. Pulse oximeter, Noninvasive blood pressure and electrocardiogram were established. Baseline pulse rate, SPO2 and blood pressure were noted. Study drug esmolol $1 \mathrm{mg} / \mathrm{kg}$ and lidocaine 1.5 $\mathrm{mg} / \mathrm{kg}$ of $10 \mathrm{~mL}$ volume were given intravenously during preoxygenation. ${ }^{11,12}$ After 5 minutes of intubation, again the blood sample for cortisol was collected. At the same time, pulse rate, diastolic blood pressure, systolic blood pressure and SPO2 were noted. Patient was maintained with nitrous oxide, oxygen, fentanyl and atracurium in titrated doses. Intraoperatively vitals were stable. After adequate attempts, patient was reserved with neostigmine and glycopyrrolate and was extubated.

In Group $\mathrm{H}$, intravenous cannula secured and blood sample for cortisol was collected. Pulse oximeter, noninvasive blood pressure, and electrocardiogram were established. Baseline pulse rate, SPO2 and blood pressure were noted. Study drug esmolol $2 \mathrm{mg} / \mathrm{kg}$ and lidocaine 2.5 $\mathrm{mg} / \mathrm{kg}$ of $10 \mathrm{~mL}$ volume were given intravenously during preoxygenation.13,14 After 5 minutes of intubation, again the blood sample for cortisol was collected. At the same time, pulse rate, diastolic blood pressure, systolic blood pressure and SPO2 were noted. Patient was maintained with nitrous oxide, oxygen, fentanyl and atracurium in titrated doses.
Intraoperatively vitals were stable. After adequate attempts, patient was reserved with neostigmine and glycopyrrolate and was extubated.

\section{Sample Size Calculation and Data Analysis}

By conducting a 20-test pre-test of 20 cases mean difference of 10.9 and standard deviation of 13.8 on serum cortisol level was obtained.

For a confidence level of $95 \%$ and a power of $85 \%$ a sample size of 18.869 (Rounded off to 20) was obtained.

The information collected regarding all the selected cases were recorded in a Master Chart. Data analysis was done with the help of computer using Epidemiological Information Package (EPI 2010) developed by Centre for Disease Control, Atlanta.

Using this software, range, frequencies, percentages, means, standard deviation, chi square and ' $p$ ' values were calculated. Kruskal Wallis Chi-square test was used to test the significance of difference between quantitative variables and Yate's Chi-square test for qualitative variables. A ' $p$ ' value less than 0.05 is taken to denote significant relationship.

\section{RESULTS}
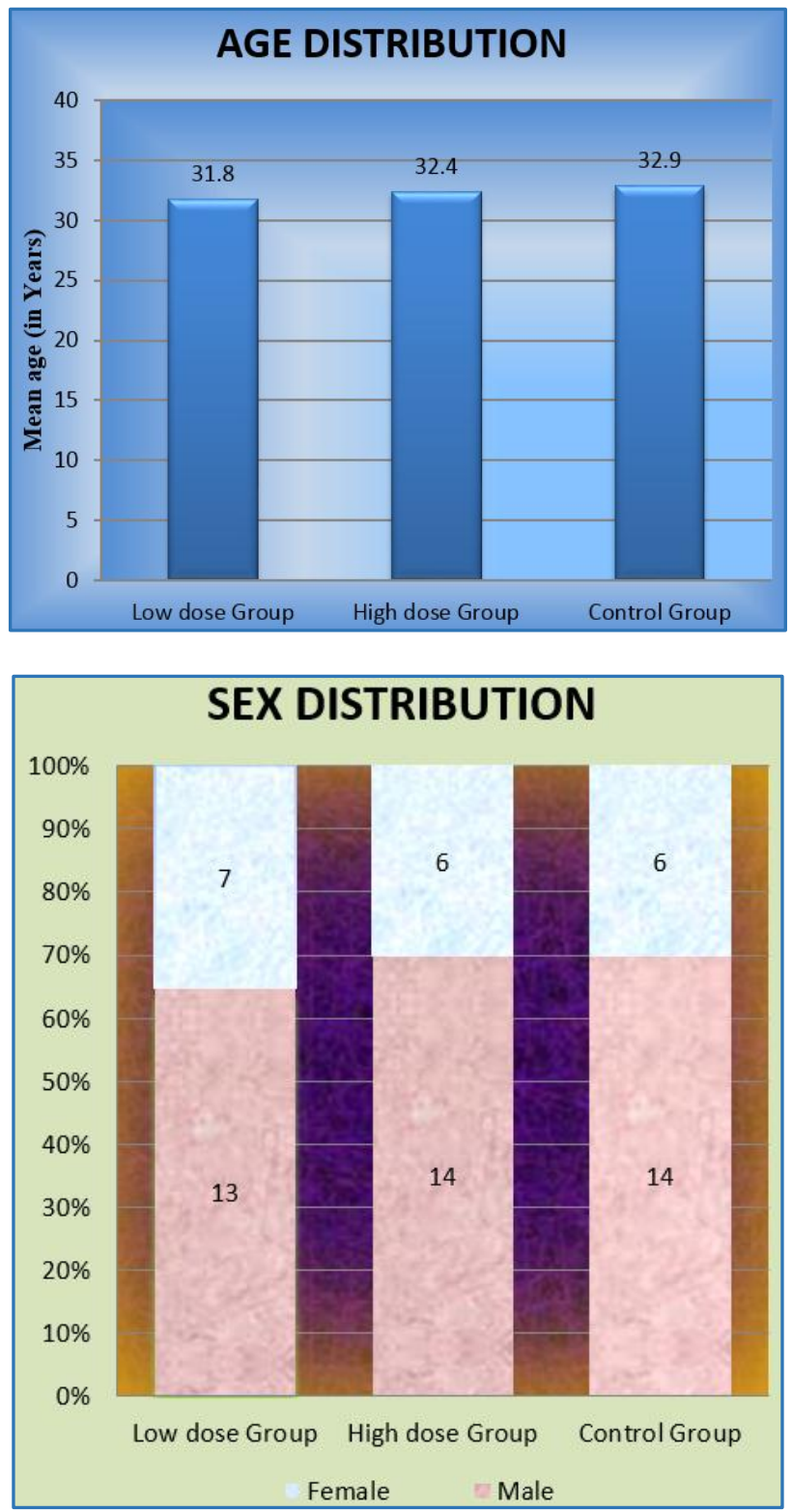

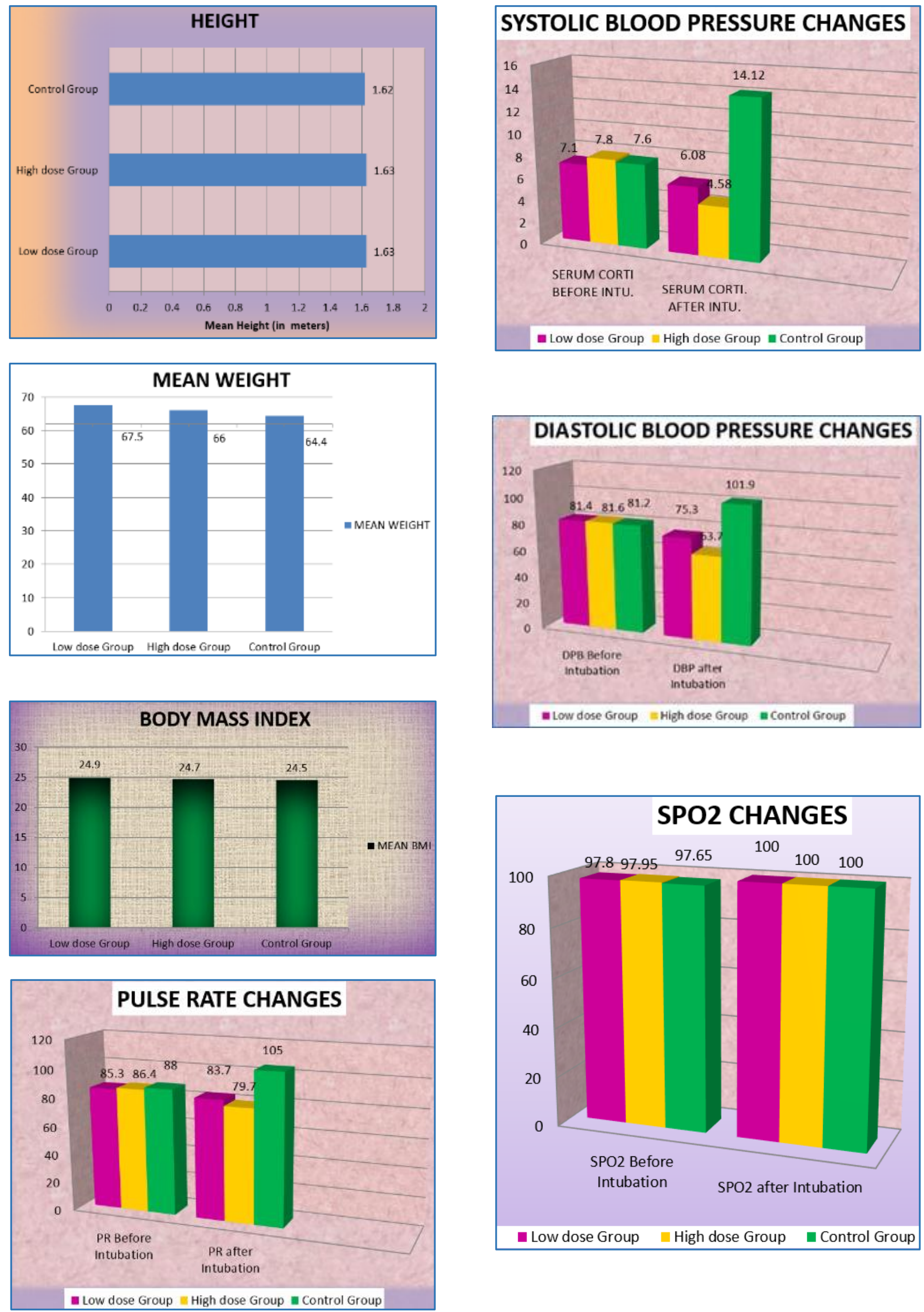


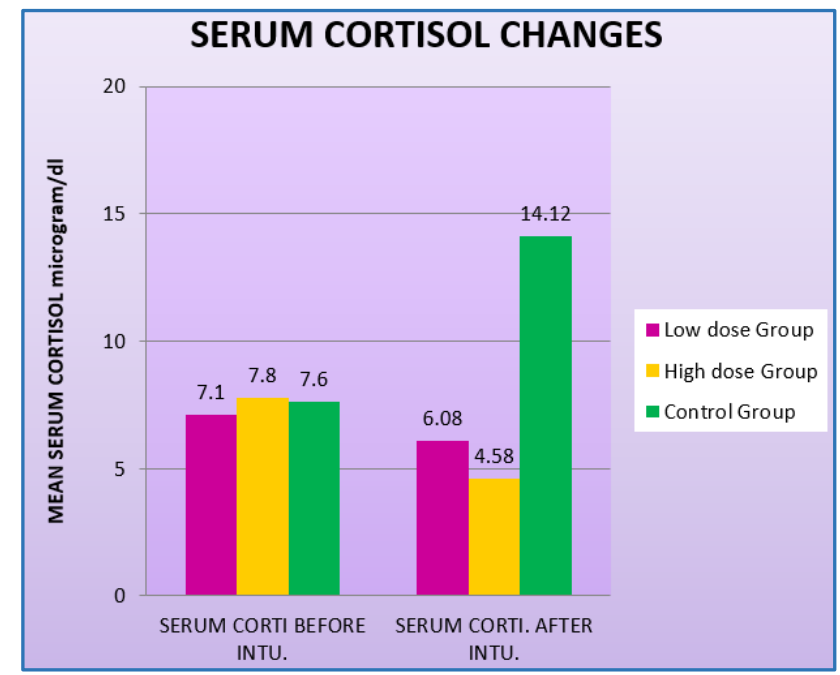

\section{Statistical Analysis}

Age, sex, height, weight and BMI were comparable between low and high dose group, low dose and control group, high dose and control group.

\section{Change in Pulse Rate}

Mean and standard deviation of pulse rate of low dose, high dose and control group were calculated before and after intubation. The difference of mean of pulse rate recorded before and after intubation were also calculated. After intubation, there is significant $(<0.0001)$ reduction in pulse rate between high dose and control group, low dose and control group and low dose and high dose group. ${ }^{15,16}$

\section{Change in Systolic Blood Pressure}

Mean and standard deviation of systolic blood pressure of low dose group, high dose group and control group were calculated before and after intubation. The difference of mean of systolic blood pressure recorded before and after intubation was also calculated. After intubation, there is significant $(<0.0001)$ reduction in systolic blood pressure between high dose and control group, low dose and control group, low dose and high dose group. ${ }^{17}$,

\section{Change in Diastolic Blood Pressure}

Mean and standard deviation of low dose, high dose and control group of systolic blood pressure were calculated before intubation and after intubation. The difference of mean of diastolic blood pressure recorded before and after intubation was also calculated. After intubation, there is significant $(<0.0001)$ reduction in diastolic blood pressure between high dose group and control group, low dose and control group, low dose group and high dose group. ${ }^{18,19}$

\section{Change in SPO2}

SPO2 monitored before and after intubation showed no significant change in all groups.

\section{Serum Cortisol Level}

Mean and standard deviation of serum cortisol level of low dose, high dose and control group were calculated before and after intubation. The difference of mean of serum cortisol level recorded before and after intubation was also calculated. After intubation, there is significant $(<0.0029)$ reduction in serum cortisol between high dose and control group, low dose and control group $(<.0001)$ and low dose and high dose group $(<0.0029)$.

Thus, high dose of esmolol and lidocaine proved to be effective in attenuating stress response occurring during intubation.

\section{DISCUSSION}

Usually, there would be increase in heart rate and blood pressure whenever intubation is attempted due to stimulation of laryngeal reflexes and psychological stress.

Many drugs have been tried to attenuate these stress responses. Drugs such as fentanyl, propofol, esmolol and lidocaine have been studied by various authors.

In the high dose group, there is a significant fall in mean heart rate than the low dose group and the control group which is comparable to the study conducted by Arti Rathore, Tanwar GL et al who compared the effect of the three different doses of esmolol and proved $150 \mathrm{mg}$ of esmolol decreased the heart rate after intubation.

In our study, there is a significant decrease in the mean cortisol level in the high dose group when compared to the low dose group and the control group which is comparable to the study conducted by Georgiou M Georgiadon, Soumpasis et al who used remifentanil and propofol to attenuate the stress response.

The decrease in heart rate and blood pressure was better when esmolol and lidocaine were used together in high dose. The fall in heart rate and blood pressure was more when compared to the response to lidocaine alone, as in the study conducted by Schumacher and Kindler showed better attenuation of haemodynamic response to laryngoscopy and intubation after giving high dose of Lidocaine and Esmolol.

There is also a study conducted by Giesecke, Hamberger and colleagues showing evidence of reduction in serum cortisol level after receiving high dose fentanyl before intubation. One more study conducted by Polo Garvin and colleagues showed similar results of decrease in cortisol level after intubation by inducing with remifentanil and propofol.

The fall in serum cortisol level is comparable with the fall noticed in other studies using propofol and fentanyl.

Thus, premedicating with $2 \mathrm{mg} / \mathrm{kg}$ of esmolol and 2.5 $\mathrm{mg} / \mathrm{kg}$ of lidocaine proved to be effective than using $1 \mathrm{mg} / \mathrm{kg}$ of esmolol and $1.5 \mathrm{mg} / \mathrm{kg}$ of lidocaine in attenuating stress response occurring during intubation.

\section{Summary}

This is a prospective randomised single blinded study involving 60 cases of ASA I and II, aged 18-65 years posted for elective general surgeries under general anaesthesia. They are allotted in 3 groups. Group $\mathrm{C}$ receiving normal saline, group L receiving low dose of lidocaine and esmolol and group $\mathrm{H}$ receiving high dose of lidocaine and esmolol. The following parameters are noted in the study period. The haemodynamic parameters such as pulse rate, systolic and diastolic blood pressure, oxygen saturation with pulse oximeter were noted before and after intubation. Along with this serum cortisol levels were monitored before and after intubation.

According to this study, there was significant reduction in pulse rate, systolic and diastolic blood pressure, and serum cortisol level after intubation in high dose group when compared to low dose group and control group. 
Thus, high dose of lidocaine and esmolol provides better attenuation of stress responses occurring during intubation, thereby decreasing the morbidity and mortality particularly in patients with poor cardiopulmonary reserve.

There were no significant side effects noted in my study.

\section{CONCLUSION}

Premedication with high doses of Esmolol and Lidocaine before intubation attenuates the stress response and thereby may decrease the morbidity and mortality in susceptible individuals.

\section{REFERENCES}

[1] Rathore A, Gupta HK, Tanwar GL, et al. Attenuation of the pressure response to laryngoscopy and endotracheal intubation with different doses of Esmolol. Ind J Anaesth 2002;46(6):449-52.

[2] Georgiou M, Georgiadou TH, Spumpasis I, et al. Endocrine stress response and haemodynamics following intubation. Propofol versus sevoflurane: A511. European Journal of Anaesthesiology 2005;22:134.

[3] Giesecke K, Hamburger B, Jarnberg PO, et al. High and low dose fentanyl anaesthesia: hormonal and metabolic response during cholecystectomy. British journal of anaesthesia 1988;61(5):575-82.

[4] Singh SP, Quadir A, Malhotra P. Comparison of esmolol and labetalol, in low does, for attenuation of sympathomimetic response to laryngoscopy and intubation. Saudi journal of anaesthesia 2010;4(3):163-8.

[5] Kindler CH, Schumacher PG, Schneider MC, et al. Effects of intravenous lidocaine and/or esmolol on haemodynamic responses to laryngoscopy and intubation: a double-blind, controlled clinical trial. Journal of Clinical Anaesthesia 1996;8(6):491-6.

[6] Feng CK, Chan KH, Liu KN, et al. A comparison of lidocaine, fentanyl, and esmolol for attenuation cardiovascular response to laryngoscopy and tracheal intubation. Acta Anaesthesiol Sin 1996;34(2):61-7.

[7] Singhal SK, Malhotra N, Kaur K, et al. Efficacy of esmolol administration at different time intervals in attenuating hemodynamic response to tracheal intubation. Indian journal of medical science 2010;64(10):468-75.

[8] Abou-Madi MN, Keszler H, Yacoub JM. Cardiovascular reactions to laryngoscopy and intubation following small and large doses of intravenous lidocaine. Can Anaesth Soc J 1977;24(1):12-9.
[9] Helfman SM, Gold MI, DeLisser EA, et al. Which drug prevents tachycardia and hypertension associated with tracheal intubation: lidocaine, fentanyl, or esmolol? Anesth Analg 1991;72(4):482-6.

[10] Parnass SM, Rothenberg DM, Kerchberger JP, et al. A single bolus dose of esmolol in the prevention of intubation induced tachycardia and hypertension in an ambulatory surgery unit. J Clin Anaesth 1990;2(4):232-7.

[11] Miller DR, Martineau RJ, Wynands JE, et al. Bolus administration of esmolol for controlling the haemodynamic response to tracheal intubation: the Canadian multicentre trial. Can J Anaesth 1991;38(7):849-58.

[12] Cucchiara RF, Benefiel DJ, Matteo RS, et al. Evaluation of esmolol in controlling heart rate and blood pressure during endotracheal intubation in patients undergoing carotid endarterectomy. Anesthesiology 1986;65(5):528-31.

[13] Hussain AM, Sultan ST. Efficacy of fentanyl and esmolol in the prevention of hemodynamic response to laryngoscopy and endotracheal intubation. J Coll Physicians Surg Pak 2005;15(8):454-7.

[14] Malde AD, Sarode V. Attenuation of the hemodynamic response to endotracheal intubation: fentanyl versus lignocaine. Int J Anesth 2007;12:1.

[15] Bakiye U, Mustafa O, Erdal G, et al. Effects of esmolol, lidocaine and fentanyl on hemodynamic response to endotracheal intubation: a comparative study. Clinical Drug Investigation 2007;27(4):269-77.

[16] Kumar A, Wahal R, Bhatia VK, et al. A study of cardiovascular response during laryngoscopy and intubation and their attenuation by ultrashort acting b-blocker esmolol. Indian J Anaesth 2002;46(2):104-6.

[17] Bostan H, Eroglu A. Comparison of clinical efficacies of fentanyl, esmolol and lidocaine in preventing hemodynamic responses to endotracheal intubation and extubation. Int J Anaesth 2012;2(1):24-8.

[18] Bruder N, Granthil C, Ortega D. Consequences and prevention methods of hemodynamic changes during laryngoscopy and intratracheal intubation. Ann Fr Anaesth Reanim 1992;11(1):57-71.

[19] Vucevic M, Purdy GM, Ellis FR. Esmolol hydrochloride for management of cardiovascular stress response to laryngoscopy tracheal intubation. British Journal of Anaesthesia 1992;68:529-30. 